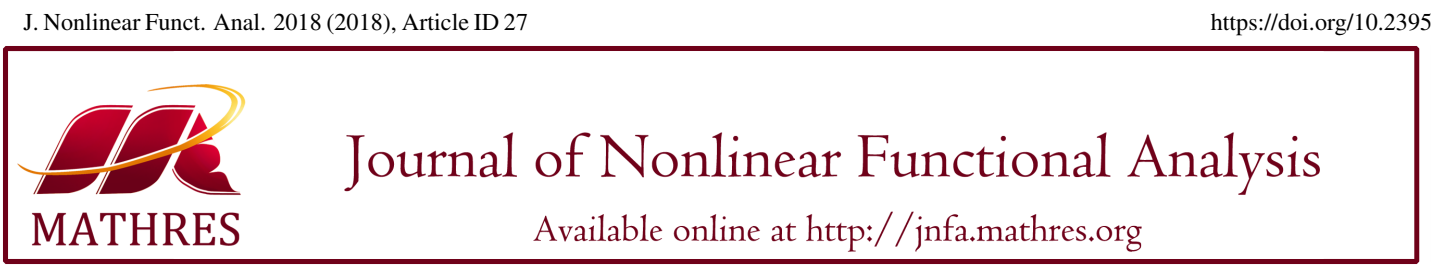

https://doi.org/10.23952/jnfa.2018.27

\title{
LINEARIZED STABILITY ANALYSIS OF CAPUTO-KATUGAMPOLA FRACTIONAL-ORDER NONLINEAR SYSTEMS
}

\author{
D. BOUCENNA ${ }^{1}$, A. BEN MAKHLOUF ${ }^{2,3, *}$, O. NAIFAR $^{4}$, A. GUEZANE-LAKOUD $^{1}$, M. A. HAMMAMI ${ }^{3}$ \\ ${ }^{1}$ AM Lab, Faculty of Sciences, Badji Mokhtar-Annaba University, P.O. Box 12, Annaba, Algeria \\ ${ }^{2}$ Department of Mathematics, College of Science, AI-Jouf University, Sakaka, Saudi Arabia \\ ${ }^{3}$ Department of Mathematics, Faculty of Sciences of Sfax, University of Sfax, Sfax, Tunisia \\ ${ }^{4}$ CEM Lab, Department of Electrical Engineering, National School of Engineering, University of Sfax, Sfax, Tunisia
}

\begin{abstract}
In this paper, a linearized asymptotic stability result for a Caputo-Katugampola fractional-order systems is described. Two applications are given to demonstrate the validity of the obtained results.

Keywords. Asymptotic stability; Caputo-Katugampola derivative; Fractional-order systems; Lotka-Volterra systems.
\end{abstract}

2010 Mathematics Subject Classification. 34A34, 34A08, 65L20.

\section{INTRODUCTION}

The integer-order calculus is inconvenient for several physical systems whose true dynamics contain fractional (non-integer) derivatives. To accurately model these systems, the fractional-order differential equations are used. For example, heat transfer systems [1], financial systems [2] and electromagnetic systems [3], have been modelled using the fractional-order calculus. Recently, the use of fractionalorder equations in the stability theory has distinctly risen $[2,4,5,6,7,8,9,10,11,12,13]$, and several works have been done in this context. There are two main methods: Lyapunov's first method as the method of linearization, the study of linear equations by means of Lyapunov exponents and theorems of linearized asymptotic stability $[6,14]$ and Lyapunov's second method: as the method of Lyapunov functions $[15,16]$.

In this paper, we propose a Lyapunov's first method for the zero solution of the following CaputoKatugampola fractional-order system of order $\alpha \in(0,1), \rho>0$ :

$$
{ }^{C} D_{t_{0}^{+}}^{\alpha, \rho} x(t)=A x(t)+f(x(t)), t \geq t_{0},
$$

\footnotetext{
${ }^{*}$ Corresponding author.

E-mail address: benmakhloufabdellatif@gmail.com (A. Ben Makhlouf).

Received February 22, 2018, Accepted August 21, 2018.
} 
where $A \in \mathbb{R}^{d \times d}$ and $f$ is a locally Lipschitz continuous function satisfying that

$$
\begin{gathered}
f(0)=0, \\
\lim _{r \longrightarrow 0} \ell_{f}(r)=0,
\end{gathered}
$$

where

$$
\ell_{f}(r):=\sup _{x, y \in B_{\mathbb{R}^{d}}(0, r)} \frac{\|f(x)-f(y)\|}{\|x-y\|} .
$$

The asymptotic stability of the zero solution of the linear Caputo-Katugampola fractional-order system:

$$
{ }^{C} D_{t_{0}^{+}}^{\alpha, \rho} x(t)=A x(t)
$$

is known to be equivalent to the spectrum $\sigma(A)$ satisfying $\sigma(A) \subset\left\{\lambda \in \mathbb{C}:|\arg (\lambda)|>\frac{\alpha \pi}{2} \mid\right\}$; see [17]. It is to be demonstrated that if the zero solution of (1.4) is asymptotically stable, then the zero solution of (1.1) is asymptotically stable which is our main result of Theorem 3.4. For the proof of the theorem, two main steps are presented. The first step consists on the transformation of the linear part to a matrix which is "very close" to a diagonal matrix. The second step describes the construction of an appropriate Lyapunov-Perron operator whose aim is to present a family of operators with the property that any solution of the nonlinear system can be interpreted as a fixed point. Finally, an application is given to show the validity of the theoretical result. For $\rho=1$, Cong et al. [6] developed the Lyapunov's first method for the zero solution of the Caputo fractional-order differential equations of order $\alpha \in(0,1)$. Our results presented in this paper mainly extend the work of Cong et al. [6].

Recently, some researches investigated the Caputo-Katugampola fractional order systems. In [18], Verma and Viswanathan investigated the Katugampola fractional calculus and fractal dimensions in which they e studied the generalized Caputo fractional integral of a continuous function of bounded variation defined on a closed bounded interval. In [19], Zeng et al. presented fractional differential equations of Caputo-Katugampola type and numerical solutions. A corresponding discretization technique was proposed. They also obtained numerical solutions discussed the convergence results. The applications of the proposed derivative is essentially illustrated by chaos [6], fuzzy logic [20] and so on. Indeed, Cong et al. [6] presented the chaos analysis and asymptotic stability of generalized Caputo fractional differential equations in which the fractional Lorenz system is described and detailed. Furthermore, Hoa, Vu and Duc [20] suggested a fuzzy fractional differential equations under the Caputo-Katugampola fractional derivative approach.

The rest of the paper is organized as follows. Some basics of the fractional-order calculus and necessary preliminaries are presented in Section 2. The linearized asymptotic stability for CaputoKatugampola fractional differential equations is given in Section 3. In Section 4, the last section, we present two applications to the Caputo-Katugampola fractional-order systems are presented in section 4 to show the efficiency of the proposed approach.

\section{PRELIMINARIES}

In this section, let us revisit some basics of the fractional calculus. We adopt the notations of the Caputo-Katugampola fractional integral and derivative from [15, 21, 22, 23]. 
Definition 2.1. (Katugampola fractional integral) Given $\alpha>0, \rho>0$ and an interval $[a, b]$ of $\mathbb{R}$, where $0<a<b$. The Katugampola fractional integral of a function $x \in L^{1}([a, b])$ is defined by

$$
I_{a^{+}}^{\alpha, \rho} u(t)=\frac{\rho^{1-\alpha}}{\Gamma(\alpha)} \int_{a}^{t} \frac{s^{\rho-1} u(s)}{\left(t^{\rho}-s^{\rho}\right)^{1-\alpha}} d s,
$$

where $\Gamma$ is the Gamma function.

Definition 2.2. (Katugampola fractional derivative) Given $0<\alpha<1, \rho>0$ and an interval $[a, b]$ of $\mathbb{R}$, where $0<a<b$. The Katugampola fractional derivative is defined by

$$
D_{a^{+}}^{\alpha, \rho} u(t)=\frac{\rho^{\alpha}}{\Gamma(1-\alpha)} t^{1-\rho} \frac{d}{d t} \int_{a}^{t} \frac{s^{\rho-1} u(s)}{\left(t^{\rho}-s^{\rho}\right)^{\alpha}} d s .
$$

Definition 2.3. (Caputo-Katugampola fractional derivative) Given $0<\alpha<1, \rho>0$ and an interval $[a, b]$ of $\mathbb{R}$, where $0<a<b$. The Caputo-Katugampola fractional derivative is defined by

$$
\begin{aligned}
{ }^{C} D_{a^{+}}^{\alpha, \rho} u(t)= & D_{a^{+}}^{\alpha, \rho}[u(t)-u(a)] \\
& =\frac{\rho^{\alpha}}{\Gamma(1-\alpha)} t^{1-\rho} \frac{d}{d t} \int_{a}^{t} \frac{s^{\rho-1}[u(s)-u(a)]}{\left(t^{\rho}-s^{\rho}\right)^{\alpha}} d s .
\end{aligned}
$$

Lemma 2.4. Let $u:\left[t_{0},+\infty\right) \rightarrow \mathbb{R}$ be a continuous function. Then, the semigroup property holds

$$
I_{a^{+}}^{\alpha, \rho} I_{a^{+}}^{\beta, \rho} u(t)=I_{a^{+}}^{\alpha+\beta, \rho} u(t), 0<\alpha, 0<\beta, 0<\rho .
$$

Lemma 2.5. The Katugampola fractional integral of $u(t)=\left(\frac{t^{\rho}-a^{\rho}}{\rho}\right)^{\beta}$ has

$$
\frac{\Gamma(\beta+1)}{\Gamma(\alpha+\beta+1)}\left(\frac{t^{\rho}-a^{\rho}}{\rho}\right)^{\beta+\alpha}, \quad-1<\beta, 0<\alpha, 0<\rho .
$$

Lemma 2.6. If $u$ is a constant, then the fractional derivative of $u$ is ${ }^{C} D_{a^{+}}^{\alpha, \rho} u(t)=0$.

Since $f$ is a locally Lipschitz continuous function, we have the existence and uniqueness of solutions of initial value problems (1.1) (see [22]). Let $x\left(t, x_{0}\right)$ denote the solution of (1.1) on its maximal interval of existence $I=\left[t_{0}, t_{\max }\left(x_{0}\right)\right)$ with $t_{0}<t_{\max }\left(x_{0}\right) \leq \infty$.

Definition 2.7. The equilibrium point $x^{*}=0$ of (1.1) is said to be

- stable if, for any $\varepsilon>0$, there is a $\delta=\delta(\varepsilon)>0$ such that, for every $\left\|x_{0}\right\| \leq \delta, t_{\max }\left(x_{0}\right)=\infty$ and

$$
\left\|x\left(t, x_{0}\right)\right\| \leq \varepsilon, \quad \forall t \geq t_{0} .
$$

- asymptotically stable if it is stable and, furthermore, there exists $c>0$ such that for every $\left\|x_{0}\right\| \leq c$, $\lim _{t \rightarrow \infty} x\left(t, x_{0}\right)=0$.

Definition 2.8. The Mittag-Leffler function with two parameters is defined as

$$
E_{\alpha, \beta}(z)=\sum_{k=0}^{+\infty} \frac{z^{k}}{\Gamma(k \alpha+\beta)}
$$

where $\alpha>0, \beta>0, z \in \mathbb{C}$. 
If $\beta=1$, then $E_{\alpha}(z)=E_{\alpha, 1}(z)$.

Proposition 2.9. [6] Let $\lambda$ be an arbitrary complex number with $\frac{\alpha \pi}{2}<|\arg \lambda| \leq \pi$. Then, the following statements hold:

(i) There exist a positive constant $M(\alpha, \lambda)$ and a positive number $t_{1}$ such that

$$
\left|t^{\alpha-1} E_{\alpha, \alpha}\left(\lambda t^{\alpha}\right)\right| \leq \frac{M(\alpha, \lambda)}{t^{\alpha+1}}, \quad \forall t>t_{1}
$$

(ii) There exists a positive constant $C(\alpha, \lambda)$ such that

$$
\sup _{t \geq 0} \int_{0}^{t}\left|(t-s)^{\alpha-1} E_{\alpha, \alpha}\left(\lambda(t-s)^{\alpha}\right)\right| d s \leq C(\alpha, \lambda) .
$$

Remark 2.10. Let $\lambda$ be an arbitrary complex number with $\frac{\alpha \pi}{2}<|\arg \lambda| \leq \pi$. Then

$$
\sup _{t \geq a} \int_{a}^{t}\left|\left(\frac{t^{\rho}-s^{\rho}}{\rho}\right)^{\alpha-1} E_{\alpha, \alpha}\left(\lambda\left(\frac{t^{\rho}-s^{\rho}}{\rho}\right)^{\alpha}\right) s^{\rho-1}\right| d s \leq C(\alpha, \lambda) .
$$

Indeed, using the change of variable $u=\frac{s^{\rho}}{\rho}$, we obtain

$$
\begin{aligned}
& \int_{a}^{t}\left|\left(\frac{t^{\rho}-s^{\rho}}{\rho}\right)^{\alpha-1} E_{\alpha, \alpha}\left(\lambda\left(\frac{t^{\rho}-s^{\rho}}{\rho}\right)^{\alpha}\right) s^{\rho-1}\right| d s \\
= & \int_{\frac{a^{\rho}}{\rho}}^{\frac{t \rho}{\rho}}\left|\left(\frac{t^{\rho}}{\rho}-u\right)^{\alpha-1} E_{\alpha, \alpha}\left(\lambda\left(\frac{t^{\rho}}{\rho}-u\right)^{\alpha}\right)\right| d u .
\end{aligned}
$$

It follows from Proposition 2.9 that

$$
\sup _{t \geq a} \int_{a}^{t}\left|\left(\frac{t^{\rho}-s^{\rho}}{\rho}\right)^{\alpha-1} E_{\alpha, \alpha}\left(\lambda\left(\frac{t^{\rho}-s^{\rho}}{\rho}\right)^{\alpha}\right) s^{\rho-1}\right| d s \leq C(\alpha, \lambda) .
$$

We consider the following fractional differential equation

$$
{ }^{C} D_{a^{+}}^{\alpha, \rho} u(t)=\lambda u(t)+h(t), u(a)=c, \quad 0<\alpha<1,0<\rho,
$$

where $h$ is a continuous function on $[a,+\infty[$. The equation can be transferred to an equivalent integral one as

$$
u(t)=u(a)+\lambda I_{a^{+}}^{\alpha, \rho} u(t)+I_{a^{+}}^{\alpha, \rho} h(t), u(a)=c .
$$

To obtain an explicit clear solution, we apply the method of the successive approximation. Set $u_{0}=$ $u(a)=c$ and

$$
u_{n+1}=u_{0}+\lambda I_{a^{+}}^{\alpha, \rho} u_{n}+I_{a^{+}}^{\alpha, \rho} h(t), 0 \leq n,
$$


It follows that

$$
\begin{gathered}
u_{1}=u_{0}+\lambda I_{a^{+}}^{\alpha, \rho} u_{0}+I_{a^{+}}^{\alpha, \rho} h(t) \\
=u_{0}+\frac{u_{0} \lambda}{\Gamma(\alpha+1)}\left(\frac{t^{\rho}-a^{\rho}}{\rho}\right)^{\alpha}+I_{a^{+}}^{\alpha, \rho} h(t) \\
=\sum_{k=0}^{1} \frac{u_{0} \lambda^{k}}{\Gamma(k \alpha+1)}\left(\frac{t^{\rho}-a^{\rho}}{\rho}\right)^{k \alpha}+I_{a^{+}}^{\alpha, \rho} h(t), \\
u_{2}=\sum_{k=0}^{2} \frac{u_{0} \lambda^{k}}{\Gamma(k \alpha+1)}\left(\frac{t^{\rho}-a^{\rho}}{\rho}\right)^{k \alpha}+\lambda I_{a^{+}}^{2 \alpha, \rho} h(t)+I_{a^{+}}^{\alpha, \rho} h(t),
\end{gathered}
$$

and

$$
\begin{aligned}
& u_{n}=u_{0} \sum_{k=0}^{n} \frac{\lambda^{k}}{\Gamma(k \alpha+1)}\left(\frac{t^{\rho}-a^{\rho}}{\rho}\right)^{k \alpha}+\sum_{k=0}^{n-1} \lambda^{k} I_{a^{+}}^{\alpha(k+1), \rho} h(t) \\
& =u_{0} \sum_{k=0}^{n} \frac{\lambda^{k}}{\Gamma(k \alpha+1)}\left(\frac{t^{\rho}-a^{\rho}}{\rho}\right)^{k \alpha} \\
& +\int_{a}^{t} \sum_{k=0}^{n-1} \lambda^{k} \frac{\rho^{1-\alpha(k+1)}}{\Gamma(\alpha k+\alpha)} \frac{s^{\rho-1}}{\left(t^{\rho}-s^{\rho}\right)^{1-\alpha(k+1)}} h(s) d s \\
& =u_{0} \sum_{k=0}^{n} \frac{\lambda^{k}}{\Gamma(k \alpha+1)}\left(\frac{t^{\rho}-a^{\rho}}{\rho}\right)^{k \alpha} \\
& +\int_{a}^{t} \sum_{k=0}^{n-1} \frac{\lambda^{k}}{\Gamma(\alpha k+\alpha)} s^{\rho-1}\left(\frac{t^{\rho}-s^{\rho}}{\rho}\right)^{\alpha(k+1)-1} h(s) d s \\
& =u_{0} \sum_{k=0}^{n} \frac{\lambda^{k}}{\Gamma(k \alpha+1)}\left(\frac{t^{\rho}-a^{\rho}}{\rho}\right)^{k \alpha} \\
& +\int_{a}^{t}\left(\frac{t^{\rho}-s^{\rho}}{\rho}\right)^{\alpha-1} s^{\rho-1} \sum_{k=0}^{n-1} \frac{\lambda^{k}}{\Gamma(\alpha k+\alpha)}\left(\frac{t^{\rho}-s^{\rho}}{\rho}\right)^{\alpha k} h(s) d s .
\end{aligned}
$$

Letting $n \rightarrow \infty$, we obtain

$$
\begin{aligned}
u(t)= & u_{0} E_{\alpha}\left(\lambda\left(\frac{t^{\rho}-a^{\rho}}{\rho}\right)^{\alpha}\right) \\
& +\int_{a}^{t}\left(\frac{t^{\rho}-s^{\rho}}{\rho}\right)^{\alpha-1} E_{\alpha, \alpha}\left(\lambda\left(\frac{t^{\rho}-s^{\rho}}{\rho}\right)^{\alpha}\right) s^{\rho-1} h(s) d s .
\end{aligned}
$$

Using Theorem 6.37, pp. 146 in [24], there exists a nonsingular matrix $T \in \mathbb{C}^{d \times d}$ transforming $A$ into the Jordan normal form, i.e.,

$$
T^{-1} A T=\operatorname{diag}\left(A_{1}, A_{2}, \ldots, A_{n}\right),
$$

where, for $i=1,2, \ldots, n, A_{i}=\lambda_{i} i d_{d_{i} \times d_{i}}+\eta_{i} N_{d_{i} \times d_{i}}$, where $\eta_{i} \in\{0,1\}, \lambda_{i} \in\left\{\widehat{\lambda_{1}}, \widehat{\lambda_{2}}, \ldots, \widehat{\lambda_{m}}\right\}$, and the nilpotent matrix $N_{d_{i} \times d_{i}}$ is given by 


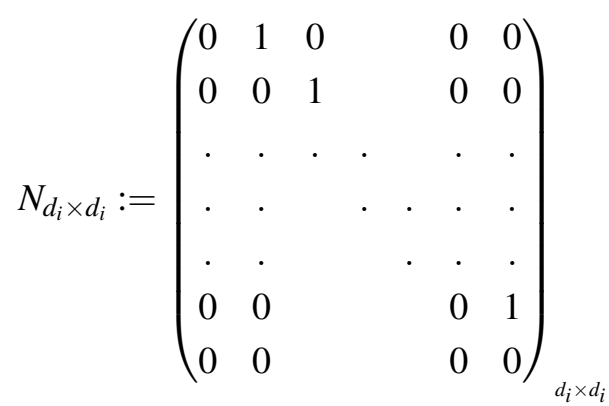

Let $\delta>0$. Using the transformation $P_{i}=\operatorname{diag}\left(1, \delta, \ldots, \delta^{d_{i}-1}\right)$, we get

$$
P_{i}^{-1} A_{i} P_{i}=\lambda_{i} i d_{d_{i} \times d_{i}}+\delta_{i} N_{d_{i} \times d_{i}},
$$

$\delta_{i} \in\{0, \delta\}$. Therefore, by the transformation $y=(T P)^{-1} x$ system (1.1) becomes

$$
{ }^{C} D_{t_{0}^{+}}^{\alpha, \rho} y(t)=\operatorname{diag}\left(J_{1}, J_{2}, \ldots, J_{n}\right) y(t)+h(y(t)),
$$

where $J_{i}=\lambda_{i} i d_{d_{i \times d_{i}}}$ for $i=1,2, \ldots, n$, and

$$
h(y)=\operatorname{diag}\left(\delta_{1} N_{d_{1} \times d_{1}}, \delta_{2} N_{d_{2} \times d_{2}}, \ldots, \delta_{n} N_{d_{n} \times d_{n}}\right) y(t)+(T P)^{-1} f(T P y) .
$$

Remark 2.11. [6] Note that the map $x \rightarrow \operatorname{diag}\left(\delta_{1} N_{d_{1} \times d_{1}}, \delta_{2} N_{d_{2} \times d_{2}}, \ldots, \delta_{n} N_{d_{n} \times d_{n}}\right) x$ is a Lipschitz continuous function with Lipschitz constant $\delta$. Thus, by (1.2), we have

$$
h(0)=0, \lim _{r \rightarrow 0} \ell_{h}(r)=\left\{\begin{array}{l}
\delta, \text { if there exists } \delta_{i}=\delta, \\
0, \text { otherwise }
\end{array}\right.
$$

Remark 2.12. [6] The type of the stability of the zero solution of equations (1.1) and (2.1) are the same.

We denote by $C_{\infty}\left(\left[t_{0},+\infty\right), \mathbb{R}^{d}\right)$ the space of all continuous functions $\xi:\left[t_{0},+\infty\right) \longrightarrow \mathbb{R}^{d}$ such that

$$
\|\xi\|_{\infty}=\sup _{t \geq t_{0}}\|\xi(t)\|<\infty .
$$

For any $x=\left(x^{1}, x^{2}, \ldots, x^{n}\right) \in \mathbb{R}^{d}=\mathbb{R}^{d_{1}} \times \ldots \times \mathbb{R}^{d_{n}}$, we define the operator

$$
\mathscr{F}_{x}: C_{\infty}\left(\left[t_{0},+\infty\right), \mathbb{R}^{d}\right) \rightarrow C_{\infty}\left(\left[t_{0},+\infty\right), \mathbb{R}^{d}\right)
$$

as follows:

$$
\left(\mathscr{F}_{x} \xi\right)(t)=\left(\left(\mathscr{F}_{x} \xi\right)^{1}(t),\left(\mathscr{F}_{x} \xi\right)^{2}(t), \ldots,\left(\mathscr{F}_{x} \xi\right)^{n}(t)\right) \quad \text { for } t \in\left[t_{0},+\infty\right),
$$

where for $i=1,2, \ldots, n$

$$
\begin{aligned}
\left(\mathscr{F}_{x} \xi\right)^{i}(t)= & E_{\alpha}\left(\left(\frac{t^{\rho}-t_{0}^{\rho}}{\rho}\right)^{\alpha} J_{i}\right) x^{i} \\
& +\int_{t_{0}}^{t}\left(\frac{t^{\rho}-s^{\rho}}{\rho}\right)^{\alpha-1} E_{\alpha, \alpha}\left(\left(\frac{t^{\rho}-s^{\rho}}{\rho}\right)^{\alpha} J_{i}\right) s^{\rho-1} h^{i}(\xi(s)) d s
\end{aligned}
$$

Remark 2.13. Let $\xi \in C_{\infty}\left(\left[t_{0},+\infty\right), \mathbb{R}^{d}\right)$. Then $\xi$ is a solution of (2.1) with $\xi\left(t_{0}\right)=x$ if and only if it is a fixed point of $\mathscr{F}_{x}$.

In the next section, we will verify that $\mathscr{F}_{x}$ is well defined. 


\section{LINEARIZED STABILITY OF CAPUTO-KATUGAMPOLA FRACTIONAL-ORDER SYSTEMS}

In this section, we study the asymptotic stability of the system (1.1).

Proposition 3.1. Assume that $\sigma(A) \subset\left\{\lambda \in \mathbb{C}:|\arg (\lambda)|>\frac{\alpha \pi}{2} \mid\right\}$. Then, there exists a constant $C(\alpha, \lambda)$ depending on $\alpha$ and $\lambda:=\left(\lambda_{1}, \lambda_{2}, \ldots, \lambda_{n}\right)$ such that, for all $x \in \mathbb{R}^{d}$ and $\xi, \widehat{\xi} \in C_{\infty}\left(\left[t_{0},+\infty\right), \mathbb{R}^{d}\right)$,

$$
\left\|\mathscr{F}_{x} \xi-\mathscr{F}_{x} \widehat{\xi}\right\|_{\infty} \leq C(\alpha, \lambda) \ell_{h}\left(\max \left(\|\xi\|_{\infty},\|\widehat{\xi}\|_{\infty}\right)\right)\|\xi-\widehat{\xi}\|_{\infty}
$$

and

$$
\begin{aligned}
\left\|\mathscr{F}_{x} \xi\right\|_{\infty} \leq & \max _{1 \leq i \leq n} \sup _{t \geq t_{0}}\left|E_{\alpha}\left(\lambda_{i}\left(\frac{t^{\rho}-t_{0}^{\rho}}{\rho}\right)^{\alpha}\right)\right|\|x\| \\
& +C(\alpha, \lambda) \ell_{h}(r)\|\xi\|_{\infty} .
\end{aligned}
$$

Proof. For $i=1,2, \ldots, n$, we have

$$
\begin{aligned}
\left\|\left(\mathscr{F}_{x} \xi\right)^{i}(t)-\left(\mathscr{F}_{x} \widehat{\xi}\right)^{i}(t)\right\| \leq & \ell_{h}\left(\max \left(\|\xi\|_{\infty},\|\widehat{\xi}\|_{\infty}\right)\right)\|\xi-\widehat{\xi}\|_{\infty} \times \\
& \int_{t_{0}}^{t}\left(\frac{t^{\rho}-s^{\rho}}{\rho}\right)^{\alpha-1}\left|E_{\alpha, \alpha}\left(\lambda_{i}\left(\frac{t^{\rho}-s^{\rho}}{\rho}\right)^{\alpha}\right)\right| s^{\rho-1} d s .
\end{aligned}
$$

It follows from Remark 2.10 that

$$
\left\|\left(\mathscr{F}_{x} \xi\right)^{i}-\left(\mathscr{F}_{x} \widehat{\xi}\right)^{i}\right\|_{\infty} \leq \ell_{h}\left(\max \left(\|\xi\|_{\infty},\|\widehat{\xi}\|_{\infty}\right)\right) C\left(\alpha, \lambda_{i}\right)\|\xi-\widehat{\xi}\|_{\infty} .
$$

Thus

$$
\left\|\mathscr{F}_{x} \xi-\mathscr{F}_{x} \hat{\xi}\right\|_{\infty} \leq C(\alpha, \lambda) \ell_{h}\left(\max \left(\|\xi\|_{\infty},\|\widehat{\xi}\|_{\infty}\right)\right)\|\xi-\widehat{\xi}\|_{\infty}
$$

where

$$
C(\alpha, \lambda)=\max \left\{C\left(\alpha, \lambda_{1}\right), C\left(\alpha, \lambda_{2}\right), \ldots, C\left(\alpha, \lambda_{n}\right)\right\} .
$$

On the other hand, for $\xi=0$, we have

$$
\left(\mathscr{F}_{x} 0\right)^{i}(t)=E_{\alpha}\left(\left(\frac{t^{\rho}-t_{0}^{\rho}}{\rho}\right)^{\alpha} J_{i}\right) x^{i} .
$$

Using (3.1), we obtain (3.2). This completes the proof.

Remark 3.2. It follows from Proposition 3.1 that $\mathscr{F}_{x}$ is well-defined.

Up to now, we have found that the Lyapunov-Perron operator is well-defined and Lipschitz continuous. Note that the Lipschitz constant $C(\alpha, \lambda)$ is independent of the constant $\delta$ which is hidden in the coefficients of system (2.1). From now on, we choose and fix the constant $\delta$ as follows $\delta:=\frac{1}{2 C(\alpha, \lambda)}$.

Lemma 3.3. Take $r>0$ such that

$$
q:=C(\alpha, \lambda) \ell_{h}(r)<1
$$

and set

$$
r^{*}=\frac{r(1-q)}{\max _{1 \leq i \leq n} \sup _{t \geq t_{0}}\left|E_{\alpha}\left(\lambda_{i}\left(\frac{t^{\rho}-t_{0}^{\rho}}{\rho}\right)^{\alpha}\right)\right|}
$$


Let $B_{C_{\infty}}(0, r):=\left\{\xi \in C_{\infty}\left(\left[t_{0},+\infty\right), \mathbb{R}^{d}\right):\|\xi\|_{\infty} \leq r\right\}$. Then, for any $x \in B_{\mathbb{R}^{d}}\left(0, r^{*}\right)$, we have $\mathscr{F}_{x}\left(B_{C_{\infty}}(0, r)\right) \subset$ $B_{C_{\infty}}(0, r)$ and

$$
\left\|\mathscr{F}_{x} \xi-\mathscr{F}_{x} \hat{\xi}\right\|_{\infty} \leq q\|\xi-\widehat{\xi}\|_{\infty}, \quad \forall \xi, \widehat{\xi} \in B_{C_{\infty}}(0, r)
$$

Proof. Let $x \in \mathbb{R}^{d}$ with $\|x\| \leq r^{*}$ and let $\xi \in B_{C_{\infty}}(0, r)$. It follows from (3.2) that

$$
\begin{aligned}
\left\|\mathscr{F}_{x} \xi\right\|_{\infty} \leq & \max _{1 \leq i \leq n} \sup _{t \geq t_{0}}\left|E_{\alpha}\left(\lambda_{i}\left(\frac{t^{\rho}-t_{0}^{\rho}}{\rho}\right)^{\alpha}\right)\right|\|x\| \\
& +C(\alpha, \lambda) \ell_{h}(r)\|\xi\|_{\infty} \\
\leq & (1-q) r+q r=r .
\end{aligned}
$$

Hence $\mathscr{F}_{x}\left(B_{C_{\infty}}(0, r)\right) \subset B_{C_{\infty}}(0, r)$. For any $x \in B_{\mathbb{R}^{d}}\left(0, r^{*}\right)$ and $\xi, \widehat{\xi} \in B_{C_{\infty}}(0, r)$, it follows from (3.1) and (3.3) that

$$
\begin{aligned}
\left\|\mathscr{F}_{x} \xi-\mathscr{F}_{x} \hat{\xi}\right\|_{\infty} & \leq C(\alpha, \lambda) \ell_{h}(r)\|\xi-\widehat{\xi}\|_{\infty} \\
& \leq q\|\xi-\widehat{\xi}\|_{\infty} .
\end{aligned}
$$

The proof is completed.

Consider system (1.1) ${ }^{C} D_{t_{0}^{+}}^{\alpha, \rho} x(t)=A x(t)+f(x(t)), t \geq t_{0}$. We now in a position to state the main result of this paper.

Theorem 3.4. Assume that $\sigma(A) \subset\left\{\lambda \in \mathbb{C}:|\arg (\lambda)|>\frac{\alpha \pi}{2} \mid\right\}$ and the nonlinear term $f$ is a locally Lipschitz continuous function satisfying (1.2). Then, the zero solution of (1.1) is asymptotically stable.

Proof. In view of Remark 2.12, it is sufficient to prove the asymptotic stability for the zero solution of system (2.1). To this end, let $r^{*}$ be defined as in (3.4). Let $x \in B_{\mathbb{R}^{d}}\left(0, r^{*}\right)$. Using the Banach fixed point theorem and Lemma 3.3, there exists a unique fixed point $\xi \in B_{C_{\infty}}(0, r)$ of $\mathscr{F}_{x}$. This point is also a solution of (2.1) with the initial condition $\xi\left(t_{0}\right)=x$. The zero solution 0 is stable, since the initial value problem for equation (2.1) has a unique solution. To complete the proof of the theorem, we have to demonstrate that the zero solution 0 is attractive. Let $x=\left(x^{1}, x^{2}, \ldots, x^{n}\right) \in B_{\mathbb{R}^{d}}\left(0, r^{*}\right)$ and $\xi(t)=\left((\xi)^{1}(t),(\xi)^{2}(t), \ldots,(\xi)^{n}(t)\right)$ be the solution of (2.1) which satisfies $\xi\left(t_{0}\right)=x$. From Lemma 3.3, we see that $\|\xi\|_{\infty} \leq r$. Let $b:=\limsup _{t \rightarrow \infty}\|\xi(t)\|$ thus $b \in[0, r]$. Let $\varepsilon>0$. Then, there exists $T(\varepsilon)>t_{0}$ such that

$$
\|\xi(t)\|<b+\varepsilon, \quad \forall t \geq T(\varepsilon)
$$


Using Proposition $2.9(i)$, we get

$$
\begin{aligned}
& \limsup _{t \rightarrow \infty}\left\|\int_{t_{0}}^{T(\varepsilon)}\left(\frac{t^{\rho}-s^{\rho}}{\rho}\right)^{\alpha-1} E_{\alpha, \alpha}\left(\lambda_{i}\left(\frac{t^{\rho}-s^{\rho}}{\rho}\right)^{\alpha}\right) s^{\rho-1} h^{i}(\xi(s)) d s\right\| \\
\leq & \max _{t \in\left[t_{0}, T(\varepsilon)\right]}\left\|h^{i}(\xi(t))\right\| \limsup _{t \rightarrow \infty} \int_{t_{0}}^{T(\varepsilon)} \frac{M\left(\alpha, \lambda_{i}\right)}{\left(\frac{t^{\rho}-s^{\rho}}{\rho}\right)^{\alpha+1}} s^{\rho-1} d s \\
\leq & \max _{t \in\left[t_{0}, T(\varepsilon)\right]}\left\|h^{i}(\xi(t))\right\| \limsup _{t \rightarrow \infty} \int_{t_{0}}^{T(\varepsilon)} \frac{\rho^{\alpha+1} M\left(\alpha, \lambda_{i}\right)}{\left(t^{\rho}-s^{\rho}\right)^{\alpha+1}} s^{\rho-1} d s \\
\leq & \max _{t \in\left[t_{0}, T(\varepsilon)\right]}\left\|h^{i}(\xi(t))\right\| \limsup _{t \rightarrow \infty} \int_{t_{0}^{\rho}}^{T(\varepsilon)^{\rho}} \frac{\rho^{\alpha} M\left(\alpha, \lambda_{i}\right)}{\left(t^{\rho}-u\right)^{\alpha+1}} d u=0 .
\end{aligned}
$$

Thus, it follows from the fact that $\xi^{i}(t)=\left(\mathscr{F}_{x} \xi\right)^{i}(t)$ and $\lim _{t \rightarrow \infty} E_{\alpha}\left(\lambda_{i}\left(\frac{t^{\rho}-t_{0}^{\rho}}{\rho}\right)^{\alpha}\right)=0$ that

$$
\begin{aligned}
\limsup _{t \rightarrow \infty}\left\|\xi^{i}(t)\right\| & =\limsup _{t \rightarrow \infty}\left\|\int_{T(\varepsilon)}^{t}\left(\frac{t^{\rho}-s^{\rho}}{\rho}\right)^{\alpha-1} E_{\alpha, \alpha}\left(\left(\frac{t^{\rho}-s^{\rho}}{\rho}\right)^{\alpha}\right) s^{\rho-1} h^{i}(\xi(s)) d s\right\| \\
& \leq \ell_{h}(r) C\left(\alpha, \lambda_{i}\right)(b+\varepsilon) .
\end{aligned}
$$

Therefore,

$$
\begin{aligned}
b & \leq \max \left\{\limsup _{t \rightarrow \infty}\left\|\xi^{1}(t)\right\|, \limsup _{t \rightarrow \infty}\left\|\xi^{2}(t)\right\|, \ldots, \limsup _{t \rightarrow \infty}\left\|\xi^{n}(t)\right\|\right\} \\
& \leq \ell_{h}(r) C(\alpha, \lambda)(b+\varepsilon) .
\end{aligned}
$$

Letting $\varepsilon \rightarrow 0$, we find

$$
b \leq \ell_{h}(r) C(\alpha, \lambda) b .
$$

From the assumption $\ell_{h}(r) C(\alpha, \lambda)<1$, we obtain that $b=0$. This ends the proof.

\section{Applications}

4.1. Fractional-order Lorenz system. To illustrate the theoretical result and to show its effectiveness, the generalized fractional-order Lorenz System is given as an example. Such system can be written as:

$$
\left\{\begin{array}{l}
{ }^{C} D_{t_{0}^{+}}^{\alpha, \rho} x_{1}(t)=a\left(x_{1}(t)-x_{2}(t)\right), \\
{ }^{C} D_{t_{0}^{+}}^{\alpha, \rho} x_{2}(t)=b x_{1}(t)-x_{1}(t) x_{3}(t)-c x_{2}(t), \\
{ }^{C} D_{t_{0}^{+}}^{\alpha, \rho} x_{3}(t)=x_{1}(t) x_{2}(t)-d x_{3}(t),
\end{array}\right.
$$

where $a, b, c$ and $d$ are parameters and $\alpha, \rho$ are the fractional-orders. It can be rewritten as

$$
{ }^{C} D_{t_{0}^{+}}^{\alpha, \rho} x(t)=A x(t)+g(x(t))
$$


where $x=\left(x_{1}, x_{2}, x_{3}\right)^{T}, A=\left(\begin{array}{ccc}a & -a & 0 \\ b & -c & 0 \\ 0 & 0 & -d\end{array}\right)$ and $g(x)=\left(\begin{array}{c}0 \\ -x_{1} x_{3} \\ x_{1} x_{2}\end{array}\right)$. Let us consider $a=-8, b=$ 26, $c=-7, d=3, \alpha=0.9$, and $\rho=1.2$ We design the linear state feedback controller as $u=B K x$ and select

$$
B=\left(\begin{array}{l}
0 \\
1 \\
0
\end{array}\right) \text { and } K=\left(\begin{array}{lll}
0 & -50 & 0
\end{array}\right)
$$

The eigenvalue of the matrix $A+B K$ are $\lambda_{1}=-2.8229, \lambda_{2}=-48.1771$ and $\lambda_{3}=-3$, which make $\left|\arg \left(\lambda_{i}\right)\right|>\frac{\alpha \pi}{2}, i \in\{1,2,3\}$. Thus, according to Theorem 3.4, the zero solution of the closed-loop system

$$
{ }^{C} D_{t_{0}^{+}}^{\alpha, \rho} x(t)=A x(t)+g(x(t))+B u(t),
$$

is asymptotically stable.

4.2. Fractional-order Lotka-Volterra system. In this subsection, we investigate the problem of the stablization by the linear feedback of the following generalized fractional-order system

$$
\left\{\begin{array}{l}
{ }^{C} D_{t_{0}^{+}}^{\alpha, \rho} x_{1}(t)=x_{1}\left(h+a x_{1}+b x_{2}\right) \\
{ }^{C} D_{t_{0}^{+}}^{\alpha, \rho} x_{2}(t)=x_{2}\left(-r+c x_{1}\right)
\end{array}\right.
$$

where the parameters $h$ and $r$ are positive. This system can be rewritten as follows

$$
{ }^{C} D_{t_{0}^{+}}^{\alpha, \rho} x(t)=A x(t)+f(x(t)),
$$

where $x=\left(x_{1}, x_{2}\right)^{T}, A=\left(\begin{array}{cc}h & 0 \\ 0 & -r\end{array}\right)$ and $f(x)=\left(\begin{array}{c}a x_{1}^{2}+b x_{1} x_{2} \\ c x_{1} x_{2}\end{array}\right)$.

We design the linear state feedback controller as $u=B K x$.The linear part of the closed-loop system is given by

$$
A+B K=\left(\begin{array}{cc}
-h & 0 \\
-2 h & -r
\end{array}\right)
$$

with $B=\left(\begin{array}{l}1 \\ 1\end{array}\right)$ and $K=\left(\begin{array}{ll}-2 h & 0\end{array}\right)$. The eigenvalue of $A+B K$ are $\lambda_{1}=-h$ and $\lambda_{2}=-r$, which make the $\left|\arg \left(\lambda_{i}\right)\right|>\frac{\alpha \pi}{2}$, for $i=1,2$. According to Theorem 3.4, the zero solution of the closed-loop system is asymptotically stable.

\section{CONCLUSION}

In this paper, we described a linearized asymptotic stability result for a Caputo-Katugampola fractionalorder system. An application is given to demonstrate the validity of the proposed results. For the proof, we proposed two main steps. The first step consists on the transformation of the linear part to a matrix. The second step describes the construction of an appropriate Lyapunov-Perron operator. Two applications are also given to show the validity of the theoretical result. 


\section{REFERENCES}

[1] S. Dadras, H.R. Momeni, A new fractional order observer design for fractional order nonlinear systems, In: Proceedings of ASME 2011 International Design Engineering Technical Conference \& Computers and Information in Engineering Conference, pp. 403-408, 2011.

[2] S. Dadras, H. R. Momeni, Fractional sliding mode observer design for a class of uncertain fractional order nonlinear systems, in: 2011 50th IEEE Conference on Decision and Control and European Control Conference (CDC-ECC), Orlando, FL, USA, pp. 6925-6930, 2011.

[3] N. Engheta, On fractional calculus and fractional multipoles in electromagnetism IEEE T. Antenn. Propag. 44 (1996), 554-566.

[4] M.A. Ansari, D. Arora, S.P. Ansari, Chaos control and synchronization of fractional order delay-varying computer virus propagation model, Math. Methods Appl. Sci. 39 (2016), 1197-1205.

[5] A. Ben Makhlouf, M A. Hammami, K. Sioud, Stability of fractional-order nonlinear systems depending on a parameter, Bull. Korean Math. Soc. 54 (2017), 1309-1321.

[6] N.D. Cong, T.S. Doan, S. Siegmund, H.T. Tuan, Linearized asymptotic stability for fractional differential equations, Electron. J. Qual. Theory Differ. Equ. 39 (2016), 1-13.

[7] S. Kumar, A. Kumar, Z. M. Odibat, A nonlinear fractional model to describe the population dynamics of two interacting species, Math. Methods Appl. Sci. 40 (2017), 4134-4148.

[8] O. Naifar, A. Ben Makhlouf, M. A. Hammami, Comments on "Lyapunov stability theorem about fractional system without and with delay" Commun. Nonlinear Sci. Numer. Simul. 30 (2016), 360-361.

[9] O. Naifar, A. Ben Makhlouf, M. A. Hammami, Comments on "Mittag-Leffler stability of fractional-order nonlinear dynamic systems [Automatica 45(8) (2009) 1965-1969]", Automatica 75 (2017), 329.

[10] I. Petras, Stability of Fractional-Order Systems with Rational Orders, arXiv:0811.4102v2, 2008.

[11] M. Rivero, S.V. Rogosin, J.A.T. Machado, J.J. Trujillo, Stability of fractional order systems, Math. Probl. Eng. 2013 (2013), Article ID 356215.

[12] M. S. Tavazoei, M. Haeri, A note on the stability of fractional order systems, Math. Comput. Simulation 79 (2009), 1566-1576.

[13] X.J. Wen, Z.M. Wu, J.G. Lu, Stability analysis of a class of nonlinear fractional-order systems, IEEE Trans. Circuits Syst. II. Express Briefs. 55 (2008), 1178-1182.

[14] N.D. Cong, T.S. Doan , S. Siegmund, H.T. Tuan, On fractional lyapunov exponent for solutions of linear fractional differential equations, Fract. Calc. Appl. Anal. 17 (2014), 285-306.

[15] D. Baleanu, G.C. Wu, S.D. Zeng, Chaos analysis and asymptotic stability of generalized Caputo fractional differential equations, Chaos, Solitons Fractals 102 (2017), 99-105.

[16] M. A. Duarte-Mermoud, N. Aguila-Camacho, J. A. Gallegos, R. Castro-Linares, Using general quadratic Lyapunov functions to prove Lyapunov uniform stability for fractional order systems, Commun. Nonlinear Sci. Numer. Simul. 22 (2015), 650-659.

[17] D. Matignon, Stability result on fractional differential equations with applications to control processing, In IMACS-SMC Proceedings, Lille, France, pp. 963-968, 1996.

[18] S. Verma, P. Viswanathan, A note on Katugampola fractional calculus and fractal dimensions, Appl. Math. Comput. 339 (2018), 220-230.

[19] S. Zeng, D. Baleanu, Y. Bai, G. Wu, Fractional differential equations of Caputo-Katugampola type and numerical solutions, Appl. Math. Comput. 315 (2017), 549-554.

[20] N.V. Hoa, H. Vu, T.M. Duc, Fuzzy fractional differential equations under Caputo-Katugampola fractional derivative approach, Fuzzy Sets and System, Doi: 10.1016/j.fss.2018.08.001.

[21] R. Almeida, A.B. Malinowska, T. Odzijewicz, Fractional differential equations with dependence on the CaputoKatugampola derivative, J. Comput. Nonlinear Dynam. 11 (2016), 061017.

[22] U. N. Katugampola, Existence and uniqueness results for a class of generalized fractional differential equations, preprint arXiv: 1411.5229.

[23] U. N. Katugampola, New approach to a generalized fractional integral, Appl. Math. Comput. 218 (2011), 860-865.

[24] G. E. Shilov, Linear algebra, Dover Publications, New York, 1977. 